\title{
ON A BOUNDARY LAYER THEORY FOR NON-NEWTONIAN FLUIDS
}

\author{
K. R. Rajagopal \\ A. S. Gupta* \\ and \\ A. S. Wineman \\ Department of Mlechanical Engineering and Applied Mechanics \\ The University of Michigan \\ Ann Arbor, MI 48109 \\ Commicated by S.I. Pai \\ (Manuscript Received December 26, 1979) \\ *Permanent address: Department of Mathematics Indian Institute of Technology, \\ Kharagour, India.
}

\section{ABSTRAC'}

Theoretical support for the existence of a boundary layer theory, similar to the classical boundary layor thoory for $1 j$ nearly viscous fluids, is offered in the case of the nonNewtonian fluid of second grade. It is also pointed out that unless cemiain assumptions are made regarding the flow, assumptions which are not alluded to in earlier work in this area, in addition to the assumptions usually made in the case of the linearly riscous fluid, the theory so developed might have inherent fias.

1. Introduction

The incompressible homogeneous Rivlin-Ericksen fluid of second grade has been studied in detail by both experimentalists and theoreticians interested in understanding the non-Newtonian behavior of fluids. The Cauchy stress $I$ and the fluid motion, in such a fluid, are assumed to be related as follows:

$$
I=-p_{\sim}+\underset{\sim}{\sim} A_{1}+\alpha_{1} A_{\sim}+\alpha_{2} A_{\sim}^{2},
$$

where

$$
\underset{\sim}{A_{1}}=\operatorname{grad} \underset{\sim}{v}+(\operatorname{grad} v)^{\top},
$$

and

$$
{\underset{\sim}{2}}_{2}=\dot{A}_{\sim 1}+A_{\sim} \operatorname{grad} \underset{\sim}{v}+(\operatorname{grad} \underset{\sim}{v})^{\top} A_{1}
$$


The $-p$ ) in equation (1.1), denotes the constitutively indeterminate spherical stress due to the constraint of incompressibility, $\mu$ is the viscosity, and $\alpha_{1}$ and $\alpha_{2}$ the normal stress moduli. The dot in equation $(1.1)_{3}$ denotes the material time derivative and $v$ denotes the velocity field. The kinematical tensors $A_{2}$ and $A_{2}$ are the Rivlin-Ericksen tensors of order 1 and 2 , respectively.

While the constitutive relation (1.1) can be considered as the second order approximation of a simple fluid, in the sense of retardation, (Coleman and Noll [1]), since the relation (1.1) is properly invariant, it could also be thought of as an exact model for some fluid. A detailed study of the thermodynamics of a fluid modeled exactly by equation (1.1) has been carried out by Dunn and Fosdick [2]. While they dealt with questions regarding the dynamic and thermodynamic stability and the uniqueness of a certain class of flows of such fluids, and provided a detailed account of the same, they did not address themselves to a variety of questions analogous to those in the theory of Newtonian fluids.

In this analysis, we would like to consider flows of fluids modeled exactly by (1.1) where inertial effects are significant. We wish to develop a boundary layer theory* similar to the classical boundary layer theory for a viscous fluid, in the case of a non-Newtonian fluid which can be modeled by equation (7.1).

It is found that the equations of motion of a second grade fluid are satisfied by an irrotational flow, very similar to the equations of motion of a Newtonian fluid. Of course, such a flow would never satisfy the boundary condition. However, that an irrotational flow satisfies the equations of motion of a fluid modeled by equation (1.1) is a remarkable property it shares with a Nevtonian fluid, from amongst the class of

Considerable attention has been paid to formulating a boundary layer theory for non-Newtonian fluids [3], [4], and [5], wherein the constitutive relation for the fluid is similar to the equation (i.1). While the authors of these papers assume the existence of a boundary layer and carry out an analysis similar to that of the classical boundary layer analysis for the NavierStokes theory, they do not offer any theoretical support as to why such a boundary layer should be expected. More importantly, they do not emphasize the assumptions which ought to be made in order to be consistent with the assumption of the existence of a boundary layer for such fluids. 
fluids of grade $n$. It can be very easily shown that the equations of motion, for instance, of the third grade fluid will not be satisfied by an irrotational flow". However, the fluids of second grade exhibit the following contrasting behavior, when compared to the Newtonian fluids. While for plane flows in the theory of Newtonjan fluids, it can be shown that the vorticity can never attain a maximum in the interior of the flow domain, no such result can be established in the theory of fluids of second grade. The above similarities and dissimilarities have relevance in establishing a boundary layer theory. Also, an important question in the theory of the fluids of form $(1.1)^{+t}$ is the kind of boundary condition which has to be prescribed, the usual no slip condition being insufficient.*

Finally, if equation (1.1) is considered to be an exact model, one could gainfully employ the results that can be obtained from the thermodynamics of such fluids. In fact, it has been shown [6] that non-compliance with the restrictions based on the thermodynamics leads to behavior which is not to be expected in fluids of rheological interest. Also, while a "slow" flow approximation could yet lead to a boundary layer theory, the assumption that the fluid model is exact el iminates the possibility of a contradiction between the constitutive assumption and the assumption of a high Reynolds number flow.

\section{Equations of Motion}

The system of field equations governing the motion of an incompressible fluid of second grade is given by

$$
\operatorname{div} T+p b=\rho \dot{v}
$$

and

$$
\text { div } \underset{\sim}{v}=0
$$

\footnotetext{
+ See Section 2 .

tf C.f. section 2 .

Such a problem does not arise in the "slow" flow approximation theory relating to equation (1.1), wherein a perturbation approach is usually employed. The usual adherence condition is found to be sufficient. However, in the general case of a fluid with memory, (e.g. viscoelastic fluids), since the stress is a non-linear function of the history of the deformation gradient, one would have to employ a boundary condition which would reflect this history.
} 
The equation $(2.1)_{2}$ is a consequence of requiring all possible motions be isochoric. We shall assume henceforth that the body force $b$ is conservative. On entering equation (1.1) in equation (2.1), we obtain after a lengthy but straight-forward manipulation, that

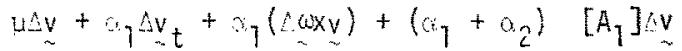

$$
\begin{aligned}
& +2 \operatorname{div}\left([\operatorname{grad} v][\operatorname{grad}]^{\top}\right)-p v_{t}-p \omega x y=\operatorname{grad} p,(2.2)_{1}
\end{aligned}
$$

where

$$
P=p-a_{1} \underset{\sim}{v} \cdot \Delta y-(1 / 4)\left(2 \alpha_{1}+a_{2}\right)\left|A_{-1}\right|^{2}+(1 / \not)|\underline{q}|^{2}+\rho \phi=(2.2)_{2}
$$

and

$$
\stackrel{\omega}{\sim} \equiv \operatorname{curl} \underset{\sim}{\sim},
$$

$\Delta$ is the Laplacian operator in three dimensions, $\underset{\sim}{v}$ denotes the partial derivative of $\unrhd$ with respec: to time, $\left|\underset{\sim}{A_{1}}\right|^{2}$ the usual trace norm of $\underset{\sim}{A_{1}}$, and where $\underset{\sim}{b} \equiv \operatorname{grad} \phi$.

If an incompressible fluid of second grade is to undergo motions which are compatible with thermodynamics in the sense that the Clausius-Duhem inequality and the assumption the the specific Helnholtz free erergy be a minimum when the fluid is locally at rest* be met, it then follows that [2],

$$
\mu \geq 0, \alpha_{1} \geq 0 \text {, and } \alpha_{1}+\alpha_{2}=0 \text {. }
$$

On making use of (2.3), one can further simplify (2.?), to the form

$$
-p{\underset{\sim}{t}}_{t}+\mu \Delta \underset{\sim}{v}+\alpha_{1} \underset{\sim}{\Delta} v_{t}+\alpha_{1}\left(\Delta \underline{\sim}^{\prime} \times \underset{\sim}{v}\right)=\operatorname{grad} P
$$

where $\underset{\sim}{\sim}$ and $P$ are as defined in $(2.2)$. On operating with the curt on both sides of the equation (2.4), one obtains that [8]:

$$
\begin{aligned}
& \left.-p \underset{\sim}{\omega}+\mu \underset{\sim}{\omega}+\alpha_{1}(\underset{\sim}{\Delta \omega})_{t}+\alpha_{1} \operatorname{curl}(\Delta \omega) v\right) \\
& -p \operatorname{curl}(\underset{\sim}{\omega} \times \underset{\sim}{v})=0
\end{aligned}
$$

\footnotetext{
$+\quad$ This of course does not preclude the possibility that for some simple fluid a second order approximation in the sense of the form (1.1) could hold with $\alpha_{1}<0$ and $\alpha_{1}+\alpha_{2} \neq 0$.

* If an incompressible fluid of second grade undergoes motion which are compatible with the Clausius-Duhem inequality, then the specific Helmholtz free energy has the form [2]:

$$
\psi=\hat{\psi}\left(\theta, A_{\sim}, A_{2}\right)=\bar{\psi}\left(\theta, A_{\sim}\right)=\bar{\psi}(\theta, 0)+\alpha_{1}\left|A_{1}\right|^{2} \text {. }
$$

In fact, for a general incompressible Rivlin-Ericksen fluid of complexity $n$, it has been established that [7]

$$
\left.\psi \equiv \hat{\psi(A,} A_{1}, \ldots A_{n}\right)=\bar{\psi}\left(\theta, A_{1}, \ldots, A_{n-1}\right) \text {. }
$$

By the statement that the s̃pecifič Helmholt frẽe enerğ of a second grade fluid be a minimum when the fluid in locally at rest, we mean

$$
\bar{\psi}(\theta, 0) \leq \bar{\psi}\left(\theta, A_{\uparrow}\right)
$$

for every traceless teñsor $A_{1}$.
} 
One observes that equation (2.5) would be satisfied by $\omega=\underset{\sim}{0}$. of course such a flow would not satisfy the boundary condition. However, the fact that $\underset{\sim}{\omega}=\stackrel{0}{0}$ satisfies (2.5) suggests a theory similar to that of the boundary layer theory for the Newtonian fluid may be appropriate. However, for a fluid of grade three which is undergoing motions compatible with thermodynamics, it can be shown that the equations of motion reduce to the following form [9]:

$$
\begin{aligned}
& \left.\mu \Delta v+\alpha \Delta v_{\tau}+\alpha_{1}(\Delta \alpha) \times v\right)+\left(\alpha_{1}+\alpha_{2}\right)\left\{A_{\sim} \Delta_{\sim} \Delta v+\right. \\
& \left.2 \operatorname{div}\left(\left[\operatorname{grad} v_{\sim}\right][\operatorname{grad} \underset{\sim}{v}]^{\top}\right)\right\}+B_{3}\left[A_{\sim}\right]\left(\operatorname{grad}\left(\left|A_{\sim}\right|^{R}\right)\right. \\
& +B_{3}\left|\underset{\sim}{A_{1}}\right|^{2} \underset{\sim}{v}-\rho \underset{\sim}{b}-\sigma v_{\sim}^{v}-\rho(\underset{\sim}{\omega} \times \underset{\sim}{v})=\operatorname{ar} a d P^{\prime} \text {. }
\end{aligned}
$$

where

$$
p^{\prime}=p-\alpha_{1} v \cdot \underset{\sim}{v} v-(1 / 4)\left(2 \alpha_{1}+\alpha_{2}\right)\left|\underset{\sim}{A_{1}}\right|^{2}+(1 / 2) p \mid \underset{\sim}{v_{1}^{\prime}}{ }^{2}+\rho \phi .
$$

It follows in this case, that an irrotational motion $\underset{\sim}{\omega}=0$, does not satisfy for instance the curl of the equation (2.6).

In fact, from a more general point of view if we write down the balance of momentum for an incompressible fluid in the absence of body forces, one obtains

$$
\dot{P} \dot{\sim}=-\operatorname{grad} P+\operatorname{div} \underset{\sim}{\tau},
$$

where $\tau$ is the deviatoric part of the stress tensor. One can easily show that in the case of a Newtonian fluid undergoing irrotational flow

$$
\operatorname{div} \tau=0
$$

so that the inviscid solution is also a solution of the Navier-Stokes equation. The physical significance of this is that, in an irrotational flow of a Newtonian fluid, the resultant force of the stresses on a closed surface is zero, the stresses themseives rot beirg zero. Also, in a perfect fluid, div $\tau=0$ trivially because there is no viscosity present. However, in the case of a non-Newtonian fluid, whatever its constitutive relation might be, it is not necessary that the resultant force due to the stresses on a closed surface be zero in the case of an irrotational flow. Hence div $\tau$ need not vanish and thus the irrotational flow need not satisfy the equations of motion.

For the rest of this section, let us restrict our attention to the plane flow of second grade fluids. In this case (2.5) reduces to

$$
\begin{gathered}
-\omega_{t}+\frac{\mu}{\rho} \Delta_{2} \omega+\frac{\alpha}{\rho}\left(\Delta_{2} \omega\right)_{t}+\frac{\alpha_{1}}{\rho}\left\{\underset{\sim}{v} \operatorname{grad}\left(\Delta_{2} \omega\right)\right\} \\
=\underset{\sim}{v} \cdot \operatorname{grad} \underset{\sim}{\omega},
\end{gathered}
$$

where $v$ is the velocity. The vorticity un this caso is given by

$$
\underset{\sim}{\omega}=\omega k,
$$


and $\Delta_{2}$ denotes the two-dimensional Laplacian operator. Let us consider the equation (2.6) in detail. If $u_{1}=0$, which by virtue of $(2.3)_{3}$ means $a_{2}=0$, then the equation (2.6) reduces to the equations of motion for the Navier-Stokes fluid. It the flow is steady, then

$$
(\mu / \rho) \Delta_{2} \omega=u \frac{\partial \omega}{\partial x}+v \frac{\partial \omega}{\partial y}
$$

where $\underset{\sim}{v}=u \underset{\sim}{i}+\underset{\sim}{j}$. It is clear form equation (2.7) that it is impossible for the vorticity to be a maximum in the interior [10]. However if $\alpha_{1} \neq 0$, in the case of steady flow, equation (2.6) reduces to

$$
(u / 0) \Delta_{2} \omega+\frac{\alpha_{1}}{\mu}\left\{u-\frac{\partial\left(\Delta_{2} \omega\right)}{\partial x}+v \frac{\partial\left(\Delta_{2} \omega\right)}{\partial y}\right\}=u \frac{\partial \omega}{\partial x}+v \frac{\partial \omega}{\partial y}
$$

The equation (2.10) does not necessarily rute out the possibility of the vorticity $\therefore$ being a maximum in the interior.

It should be noted that in shear flows of a fluid of second grade, normal stress differences arise in addition to tangential forces. Thus, if a boundary layer is to exist in flows of such fluids, it is necessary that not only the ratio of the inertial forces to the forces due to the tangential stresses be large, but also the ratio of the inertial forces to the forces due to the normal stresses should be large. In the case of a Newtonian fluid, the condition that the inertial forces are much larger than the forces due to the tangential stresses implies that the Reynolds number Re (based on a characteristic length scale in the flow) should be very large. In a viscoelastic fluid, such as a second grade fluid, the ratio of the forces due to the normal stresses to the inertia forces is given by the ratio of the non-dimensional parameter We and the Rcynolds number Re where

$$
\operatorname{Re}=\frac{\rho V_{\ell}}{\mu} \text {, we }=\frac{\alpha_{1} V}{\mu \ell}
$$

In the above definition (2.11), V. l, $\mu, q_{\text {and }}$, clenote a characteristic velocity, a characteristic length, the viscosity, the normal stress modulus and the density, respectively. Now, it is well-known that in a Newtonian fluid, the boundary layer theory is valid when Re $>1$, so that the flow is everywhere inviscid except in a thin layer near the boundary. In a visco elastic fiuid, one would therefore expect such a theory to hold if

$$
\operatorname{Re} \gg 1 \text { and Re/We }>1 \text {, }
$$

or equivaisatly

$$
\text { Re }>\max (1, \mathrm{We})
$$

Since Re can be thought of as the ratio of two length scales $\ell$ and $(\mu / p V)$, it follows that the boundary layer theory in a Newtonian fluid would hold in the 
presence of two disparate length scales. The inequality (2.12) however suggests that in a second grade fluid more than two disparate length scales must exist to warrant a boundary layer. It is not inconceivable, therefore, to have a boundary ijyer with a two-deck structure such that in one deck the tangential forces and the inertial forces are of the same order, while in the other deck the inertia and pressure forces balance the forces due to the normal stresses. Thus in adfition to a conventional viscous boundary layer, we may have an 'elastic' boundary layer within which the normal stresses are large. Comparison of the inertia forces and the forces due to the normes stresses in a two dimensional boundary laye:r of such a fluid gives the elastic boundary layer thickness $\delta_{E}$ as

$$
\delta_{E}-0\left(\left(^{\alpha} 1 / \rho\right)^{1 / 2}\right)
$$

Clearly $j_{E} / \ell$ is very small in view of the second inequality in (2.12). Thus, when (2.12) holds, a thin boundary layer with a two-deck structure exists with an effectively inviscid flow outside. If the number we is $0(1)$, the normal stress effects would not be important and we retrieve the conventional viscous boundary layer.

\section{Vimensional Analysis}

In the Navier-Stokes theory, since the terms involving $\alpha_{1}$ do not appear in the equations of motion, the basic non-linearity is due to the accelerating term. Howiver, for fluids of second grade, non-linearity due to terms involving $\alpha_{1}$ appear. To determine the relative importance of these non-linearities, we shall proceed to non-dimensionalize the equation (2.5) in the manner outlined in [8]. Introducing a characteristic length $\ell$, and a characteristic speed $V$, we obtain a dimensionless space co-ordinate and velocity

$$
\underline{x}^{*}=\underset{\sim}{x} / l, \underset{v}{v}=\underset{\sim}{v} / V
$$

When the coefficients $\mu$ and $\alpha_{1}$ are both non-zero, we obtain from (2.5), that

$$
\begin{aligned}
& -\operatorname{Re} \omega^{\star}+\Delta^{*} \underset{\sim}{*}+\frac{\operatorname{Re}}{\Gamma}\left(\Delta^{*} \underset{\sim}{*}\right)_{t}+\frac{\operatorname{Re}}{\Gamma} \operatorname{curl}{ }^{*}\left(\Delta^{*} \omega_{\sim}^{*} \underset{\sim}{*}\right) \\
& -\operatorname{Re} \operatorname{cur} 1 *\left(\omega^{*} \times \sim^{*}\right)=0
\end{aligned}
$$

where the Reynolds number Re and the absorption number $\Gamma$ are given through 


$$
\operatorname{Re} \equiv \frac{\rho V \ell}{\mu}, \Gamma \equiv \frac{\rho \ell<}{\alpha_{1}} \text {, }
$$

Where ${\underset{\sim}{*}}^{*}=$ curl $v_{\sim}^{*}$, and where the operators $\Delta^{*}$ and curl* are the dimensionless ccunterparts of $\Delta$ and curl. It should be clear from (3.2) that, even if $\alpha_{1}$ is considered to be small, the ratio $R / \Gamma$ could be large. Of course, since $\alpha_{1}=-\alpha_{2}$, by virture of equation (2.3) 3 , we see that neglecting terms involving $\alpha_{1}$ would imply that we are in effect considering a Navier-Stokes fluid. More importantly, since $R / T$ multiplies terms involving the highest derivatives in equation (3.1). one would obtain a singular perturbation problem for small $R / T$ and hence dropping the terms involving a might lead to erroneous rasults. Thus, the boundary layer due to the flow of a second grade fluid would be different from that of the Navier-Stokes fluid, contrary to the observations of Frater [12], due to the termis involving the coefficient $\star_{R} / \Gamma$. That the terms involving $\alpha_{1}$ can considerabiy change the character of the diffusion of velocity and vorticity from a boundary has been already pointed out by Truesdell [13].

\section{Conclusion}

Having establisheda motivation for expecting a boundary layer for fluids of second grade, namely that $\underset{\sim}{\omega}=0$ satisfies the equations of motion of a second grade fluid, and having outlined the limitations and tacit assumptions which ought to be made so that we might have a consistent theory, we could go ahead and obtain the boundary layer equations as outlined in [4], [5]. However, it is important to realize that there could be inherent inconsistensies in formulating boundary layer theories for general non-Newtonian fluids.

While [4] for instance points out that earlier work in the area of boundary layer theories for non-Newtonian fluids do not justify boundary layer approximations, it itself fails to justify the presence of a boundary layer for such non-Newtonian fluids.

Acknowledgement: The authors would like to thank Professor C. S. Yih for the numerous discussions which one of the authors(K. R. R.) had with him.

* The non-dimensional number defined through $(3.2)_{2}$ is essentially the same as that obtained by walters [11]. It follows from equations (2.11) and (3.2) that, $\Gamma=R e /$ We. Thus, it follows from equation (2.12) that if a boundary layer theory is to hold $\Gamma \gg 1$. This however does not imply that the ratio $R e / \Gamma$ can be neglected. In fact it would be erroneous to neglect the terms involving $\mathrm{Re} / \Gamma$ for we would then be essentially ignoring the non-Newtonian nature of the fluid.

** Walters [11] makes a similar observation. However, since he views the model as an approximation, he has to contend with justifications for neglecting higher order terms which appear in his equations of motion. 


\section{Bibliography}

[1] B. D. Coleman and W. Noll, "An Approximation Theorem for Functionals, with Applications in Continuum Mechanics," Arch. Ratl. Mech. Anal . 6, 355-370 (1960).

[2] J. E. Dunn and R. L. Fosdick, "Thermodynamics, Stability and Boundedners of Fluids of Complexity 2 and Fluids of Second Grade," Arch. Ratl. Mech. Anal. 3. 191-252 (1974).

[3] A. C. Srivastava, "The Flow of a Non-Newtonian Liquid Near a Stagnation Point," ZAMP, 9 , 80-84, (1958).

[4] P. L. Bhatnagar, "On Two-Dimensional Boundary Layer in Non-Newtonian Fluids with Constant Coefficients of Viscosity and Cross-Viscosity," Proc. Ind. Acad. Sci.

[5] G. K. Rajeswari and S. L. Rathna, "Flow of a Particular Class of NonNewtonian Visco-Elastic and Visco-Inelastic Fluids Near a Stagnation Point," ZAMP, 13, 43-57 (1952).

[6] R. L. Fosdick and K. R. Rajagopal, "Anomalous Features in the Model of "Second Order Fluids" Arch. Ratl. Anal (In Press).

[7] K. R. Rajagopal, "Thermodynamics and Stability of Non-Newtonian Fluids," Developments in Mechanics Vol. 10, Proceedings of the 16th Midwestern Mechanics Conference, (1979).

[8] R. L. Fosdick and K. R. Rajagopal, "Uniqueness and Drag for Fluids of Second Grade in Steady Motion," Intl. J. Non-Linear Mechanics, 13, $131-137$ (1978).

[9] R. L. Fosdick and K. R. Rajagopal, "Thermodynamics and Stability of Fluids of Grade Three," Proc. Royal Society, London (In Press).

[10] M. H. Protter and H. F. Weinberger, Maximum Principles in Differential Equations, Prentice-Hall, Englemoods-Cliff, N.J., (1967).

[11] K. Walters, "On a Boundary-Layer Controversy," ZAMP, 21 276-231 (1977).

[12] K. R. Frater, "A Boundary-Layer in an Elastico-Viscous Fluid, Fluid," ZAMP, 20, $712-721(1969)$.

[13] C. Truesde11, "The Natural Time of a Viscoelastic Fluid, Its Significance and Heasurement," Physics of Fluids," Z, 1134-1142 (1964).

[14] D. W. Beard and K. Walters, "Elasto-Viscous Boundary Layer Flows." Proc. Camb. Phil-Soc, 60, 667-674 (1964). 